\title{
Myocardial Infarction and Three-Vessel Coronary Artery Disease as Presenting Features of Granulomatosis with Polyangiitis: A Case Report with Review of Literature
}

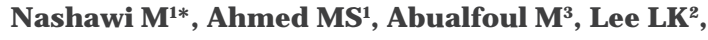
Ghali $\mathrm{A}^{\mathbf{1}}$ and Chilton $\mathrm{RJ}^{\mathbf{1}}$

${ }^{1}$ Department of Medicine-Cardiology, University of Texas Health Science Center at San Antonio, USA

${ }^{2}$ Department of Internal Medicine, The University of Texas Health Science Center, USA

${ }^{3}$ Department of Internal Medicine, Methodist Dallas Medical Center, USA

*Corresponding author: Mouhamed Nashawi, Department of Medicine-Cardiology, University of Texas Health Science Center at San Antonio, 7703 Floyd Curl Dr., San Antonio, TX, 78229-3900, USA

Received: March 08, 2021; Accepted: March 26, 2021; Published: April 02, 2021

\begin{abstract}
Granulomatosis with Polyangiitis (GPA) is a systemic, autoimmune disorder characterized by inflammatory insult and granulomatous processes in small and medium-sized vessels leading to various clinical presentations from underlying vasculitis. Underlying such inflammatory cascade is the overactivity of Antineutrophil Cytoplasmic Antibodies (c-ANCA) targeting serum Proteinase 3 (PR3), whose aberrant targeting classically modulates molecular signaling pathways leading to clinical manifestations of the Ear, Nose and Throat (ENT), in addition to renal impairment. Peripheral vessel involvement (i.e. limb vasculature) is not generally associated with GPA. With the exception of seldom reports in the literature, it is rare for GPA to present with coronary artery involvement. Moreover, reports of multi-vessel disease (e.g. triple-vessel disease) with GPA warranting Coronary Artery Bypass Graft (CABG) are lacking in such accounts. The latter with preceding iliac artery claudication makes such a presentation of GPA exceptionally novel and warrants contextual commentary regarding inflammation and Coronary Artery Disease (CAD). We report the case of a 55-year-old Caucasian male presenting with a 2 years history of right-sided groin cramping and an acute one-week history of claudication in the same area. After advised to follow up as an outpatient, this patient returned shortly thereafter to an acute care setting with hemoptysis and myocardial infarction worked up for GPA and triple-vessel disease. The patient was subsequently treated with immunosuppressive pharmacotherapy prior to CABG. We conducted a review of the literature underpinned in clinical and translational biology with a focus on the salient inflammatory pathways featured in both coronary artery disease and GPA.
\end{abstract}

Keywords: Vasculitis; Coronary; Myocardial infarction; Multi-vessel disease; Polyangiitis; Granulomatosis; Pr3

\section{Introduction}

A significant proportion of triple-vessel disease cases can be attributed to Coronary Artery Disease (CAD) [1]. Underlying severe CAD is coronary atherosclerosis and a complex, multifactorial pathologic orchestra marked initially by the accumulation of LowDensity Lipoprotein (LDL) within the subendothelial space of affected vessels, which subsequently undergoes oxidation [2,3]. This Oxidized Low-Density Lipoprotein (oxLDL) stimulates macrophage infiltration, and the uptake of oxLDL results in the formation of foam cells, or the so called infamous "fatty streak" observable under microscopy. The degradation of foam cells results in the egress of their contents, which induce a cascade of smooth cell proliferation and an inflammatory cascade culminating in a fibroinflammatory lipid plaque, called an atheroma [4,5]. This focal thickening of the arterial tunica intima hampers vessel compliance in addition to promoting a stenotic phenotype [6]. Coronary vessel occlusion may lead to inadequate myocardial perfusion and subsequent angina if anatomical restriction is significant enough to warrant a physiologically significant dearth in myocardial oxygenation [7]. One notable consequence of this resultant ischemia is cellular death of cardiomyocytes in the setting of increased oxygen demand. In the context of symptomatic CAD, compensatory physiologic processes are insufficient to provide adequate perfusion to the myocardium and may invoke a Myocardial Infarction (MI). Moreover, the lesions accrued at the commencement of vascular insult results in vessel stiffness, which promotes a state of hypertension. The mechanical forces associated with pernicious blood flow dynamics may cause plaque disruption and subsequent thrombosis in this setting. Moreover, expansion of thrombi without complete dislodgement may in itself additionally obstruction myocardial perfusion and induce MI.

Systemic inflammatory syndromes, such as the family of vasculitides, have received increased credence regarding their role as a nidus or provocateur of coronary artery disease due to aberrant signaling pathways or repeated degranulation of cytotoxic products from the leukocytes associated with these syndromes [8]. While multifarious in their pathophysiology, these conditions share a central theme of triggering bouts of inflammation that overlap with the same mediators that participate in pathologic plaque formation [9]. Substantively, every stage of atherosclerosis is 
characterized by an inflammatory process. Even more interesting is that through inflammation of the vasculature, vasculopathies such as Granulomatosis with Polyangiitis (GPA) have the potential to disrupt the structural and physiologic architecture of the vascular endothelium the most preliminary event in pathologic cholesterol deposition. Within the literature, Kawasaki Disease and Takayasu's arteritis are the classic vasculopathies associated with CAD [10-13]. However, there has been recent evidence that show that vasculitides not typically associated with CAD may also exhibit coronary artery complications ranging from mild fatty deposition to fulminant multivessel disease [14,15]. There is evidence that supports GPA as a trigger for these pathologic events through autoimmune provocation. In the case of GPA, it is also believed that Antineutrophil Cytoplasmic Antibodies (c-ANCA) promote neutrophil adherence to vascular endothelium with subsequent degranulation of cytotoxic contents serving as the focus for vasculitis, vessel insult, and subsequent cholesterol accumulation [16].

Although extremely rare per current reviews of the literature, mindfulness of the association between vasculitides such as GPA and heart disease are can be critical in delineating the clinical manifestations of patients with MI, CAD, and recent history of vasculopathy [17]. We report the case of NSTEMI and triple-vessel CAD in a middle-aged, adult male with a recent history of lower limb claudication, later worked up for GPA. Given the rarity of GPA to present with coronary involvement in the literature, reports of these encounters are useful in informing clinicians while holding value in guiding management of vasculitis with concomitant Cardiovascular Disease (CVD). Moreover, a commentary outlining the mutual pathways in GPA and CAD offers an opportunity to shape perspectives regarding autoimmune conditions and the cardiovascular disease risk associated with them by placing inflammation in the spotlight.

\section{Case Presentation}

A 55-year-old male with no significant past medical history arrived at the emergency department due to a 1 week history of sharp pain in his groin and buttocks region that worsened at night and when laying down, consistent with a picture of claudication. These episodes of sharp pain were substantial enough to cause the patient to awaken the patient from sleep. Of note, he works as a skilled laborer in the automotive industry and has reported intermittent cramping also in his groin and buttocks with radiation from his right thigh down to his calf that he attributed to work-related overuse injuries. The patient reported that cramping episodes were more frequent and severe in nature with physical exertion, prompting temporary cessation of activity until the pain subsided. The remainder of a review of systems was null for significant clinical findings. Initially, the patient used over-the-counter Nonsteroidal Anti-Inflammatory (NSAIDs) agents as needed to alleviate his pain, but they no longer were effective per the patient. Upon arrival, the patient was afebrile and hemodynamically stable with the only pertinent physical exam findings elicited being tenderness to palpation of the right lower quadrant of the abdomen. A query of past medical and surgical history was nonsignificant, as the patient has not sought medical counsel of any kind in roughly 20 years. Socially, the patient affirmed intermittent tobacco and cannabis use (quit tobacco roughly 12 years ago), and informed providers of a remote history of alcohol use disorder with complete sobriety reached over 20 years ago. His family history was unremarkable.

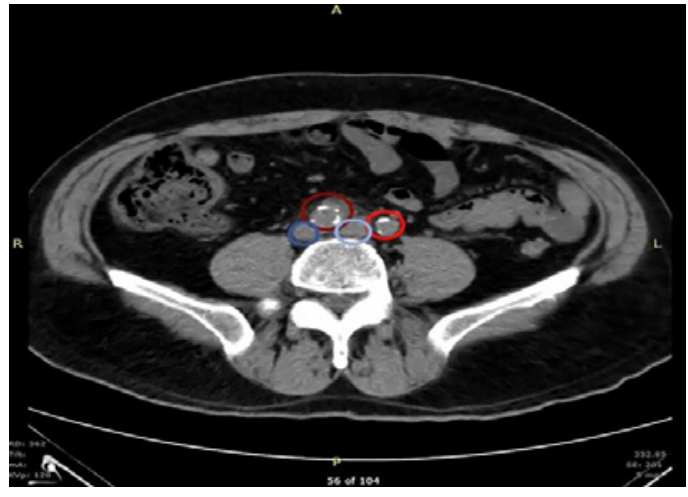

Figure 1: CT Angiogram of Chest upon Presentation. Dark Red-Right lliac Artery, Dark Blue-Right Iliac Vein, Light Red-Left lliac Artery, Light Blue-Left Iliac Vein.

Computed Tomography Angiogram (CTA) with contrast of the abdominal aorta in addition to a CT study of the abdomen and pelvis without contrast were ordered to appraise the anatomy of the inciting limb. Appraisal of this image was significant for illdefined non-enhancing attenuation of the soft tissue surrounding the proximal right iliac artery just distal to the aortic bifurcation, without extravasation or extraluminal contrast deposition. Such findings suggest inflammatory changes about the proximal right iliac artery with prevailing diagnoses being focal vasculitis versus retroperitoneal fibrosis (Figure 1). Given clinical stability of and chronicity of symptoms without emergent aggravation, it was deemed that there was no indication for acute surgical intervention. It was decided that it would be appropriate for the patient to follow-up as an outpatient with vascular surgery pending primary care clearance.

Thirteen days later, this patient returned to the emergency department without establishing any formal visitation with any medical providers for interval period surgical evaluation. He presented with coughing, shortness of breath, tachypnea (respiratory rate ranging from $26-40$ breaths per minute), diaphoresis, diffuse angina that started centrally and radiated to his left shoulder and jaw, and hemoptysis. The initial troponin levels of the patient were of $2.71 \mathrm{ng} / \mathrm{mL}$, peaking to a maximus of $8.99 \mathrm{ng} / \mathrm{mL}$ within the interim. Associated with the cough was an intermittent, sensation of "burning" chest pain that he rated as a 7/10. Per the patient, he was able to produce roughly one teaspoon's worth of bright red blood with cough five to ten times per day in the days leading up to his presentation to the emergency department. This clinical spectrum was also within the context of an unintentional weight loss of roughly $10-15$ pounds in the last 40 days.

His presentation was notable for hemodynamically stable parameters and afebrile status upon initial assessment. Physical exam was notable for inflammation and redness of the joints surrounding the knees and wrists, without edema of the lower extremities. This is consistent with the patient's report of exacerbated swelling of knee, wrist, and hand joints for the past two weeks. An obtained Electrocardiogram (ECG) showed left atrial abnormalities and ST depressions in the inferolateral leads measuring roughly $1 \mathrm{~mm}$ in depth consistent with non-ST segment Elevation Myocardial Infarction (NSTEMI) (Figure 2). The patient was given $321 \mathrm{mg}$ 

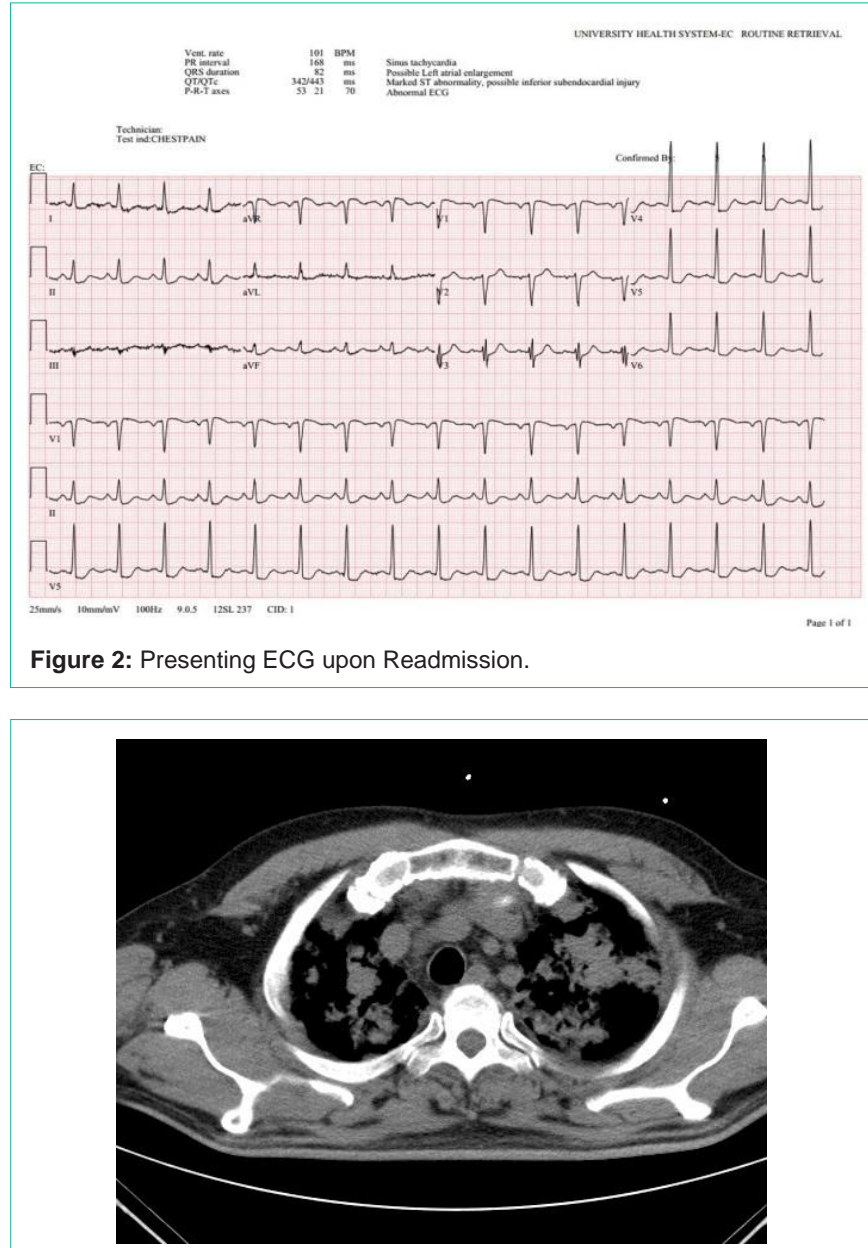

Figure 3: CT Chest showing Inflammatory Infiltrates In Lieu of Pulmonary Embolism.

of Aspirin and a low-dose statin in the emergency room with a recommendation to hold beta blockers, heparin, and clopidogrel given blood loss via hemoptysis. The presentation of hemoptysis led to a concern for potential pulmonary emboli, which were ruled out with non-significant clinical exam findings supplemented with a CT of the chest, which did however show diffuse inflammatory infiltrates (Figure 3). The latter visual phenomena were then subsequently found to be diffuse alveolar hemorrhages. Subsequent workup for infectious etiology of hemoptysis including sputum acid-fast bacilli, blood culture, legionella sputum, HIV, fungal workup, and cryptococcus were negative. Moreover, the patient denied recent fevers, chills, exposure to known or perceived sick contacts, recent travel, or a possibility for zoonotic exposure.

At this point, Inflammatory and autoimmune entities started to prevail as foremost diagnoses within the differential given exhaustive testing for infectious etiology. In reference to the previous inflammatory changes noted earlier along the right iliac artery, the prospect of underlying vasculitis started to gain prominence. Subsequently, a diagnosis of C-ANCA vasculitis with positive serum Proteinase 3 (PR3) was found, with PR3 levels measuring 112.3 Units (institutional reference range, [0.00-21.00 Units]). At this point, the previous maximum troponin of $8.99 \mathrm{ng} / \mathrm{mL}$ was followed by three

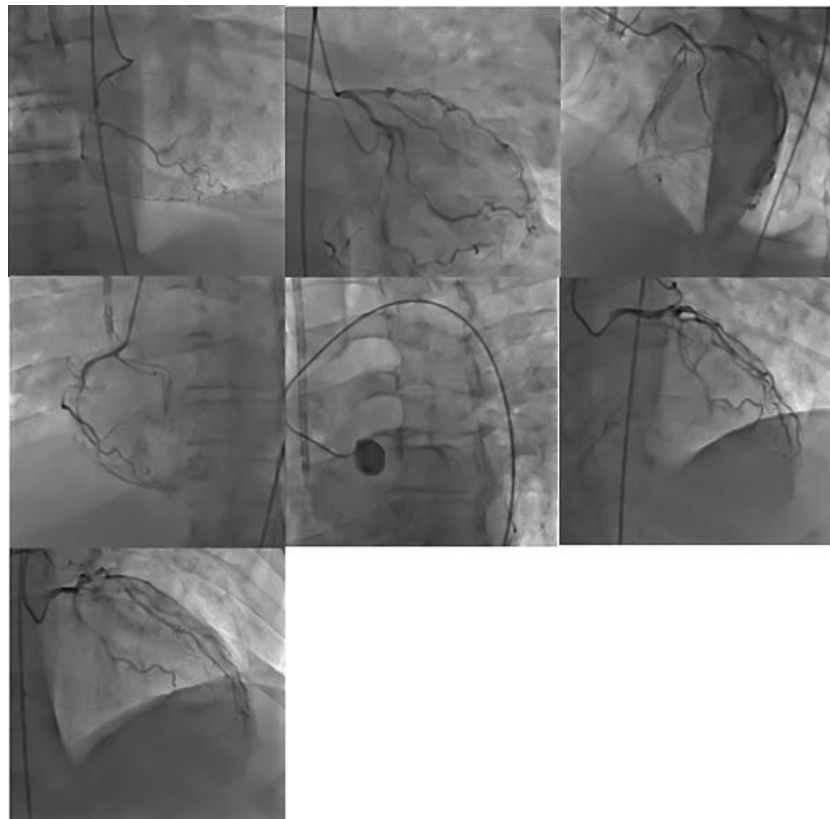

Figure 4: Catheterization Profile Showing Triple-Vessel Disease.

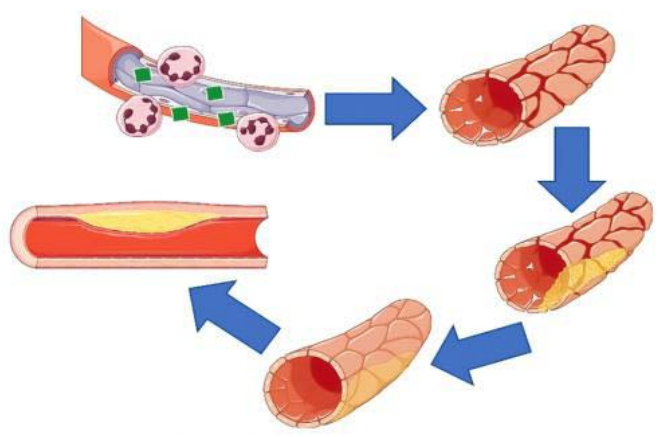

Figure 5: Influence of Inflammatory Sequelae on Atheroma formation Adapted from Servier Medical Art by Servier, with licensure under a Creative Commons Attribution 3.0 Unported License.

subsequent descending troponin values of $6.55,3.77$, and $2.94 \mathrm{ng} /$ $\mathrm{mL}$. Given the resolution of hemoptysis and concern of underlying myocardial injury warranting admission, the decision to prepare the patient for coronary catheterization was pursued. The findings of the studies revealed two large obtuse marginal branches of the Left Circumflex Artery (LCX) that exhibited 70\% stenosis each. Studies of the Right Coronary Artery (RCA) revealed dominant status, with $40 \%$ stenosis to the proximal portions, roughly $99 \%$ stenosis in the middle components, followed by Complete Total Occlusion (CTO). The most proximal portion (D1) of the Left Anterior Descending Artery (LAD) was $95 \%$ stenotic with the main artery exhibiting CTO. The following coronary audit is consistent with triple-vessel disease and representative images of the catheterization (Figure 4).

Given elevated serum PR3, hemoptysis, and vasculitis, a diagnosis of Granulomatosis with Polyangiitis (GPA) was made by rheumatology consulting services. The decision to start plasmapheresis to attenuate this patient's inflammatory status and hemoptysis was initiated with a successful transient resolution of 
symptoms. Subsequently, the patient was started on corticosteroids to mitigate inflammatory hyperreactivity. It was determined that triple-vessel disease precluded the patient from receiving minimally invasive interventional approaches to restore myocardial perfusion by interventional cardiology, and the decision to consult cardiothoracic surgery for a Coronary Artery Bypass Graft (CABG) was made. It was assessed that the patient was currently a high-bleed risk given recent hemoptysis, prolonged inflammatory history inflicting friable vasculature, and a recent corticosteroid regimen mitigating adequate wound healing. Medical management of heart disease with guideline-directed medical therapy and an eight-month course of cyclophosphamide and prednisone (which was tapered leading up to the procedure) allowed the patient to have a more tractable risk profile prior to surgical intervention. Subsequent CABG was successful with echocardiogram (ejection fraction $\sim 55 \%$ ) revealing a lack of valvular disease and adequate biventricular function. After rehabilitation, the patient subsequently noted marked improvement of angina, resolution of pillow orthopnea, and lack of dyspnea upon exertion relative to baseline standards with satisfaction in quality of life per the patient.

\section{Discussion}

\section{Background}

The etiology of GPA has not been well elucidated and extensive studies have failed to show a connection between this type of vasculitis and specific genetic associations, namely Human Leukocyte Antigen (HLA) profiles [18]. The yearly hospitalization rates for complicated episodes of granulomatosis with polyangiitis range from roughly 5.1 to 6.3 per 1 million persons in the United States [19]. The most well studied environmental insult implicated in presumed GPA exacerbations in susceptible patients has been silica [20]. The degree of silica exposure that this patient may have been exposed to given his profession as a skilled laborer in the automotive industry is a possible factor in assessing his epidemiological risk profile, but quantifying its influence and indexing such exposure to already scant evidence makes for a pursuit of indeterminate viability. Presenting symptoms during an acute flair may be very generalized, such as malaise, fatigue, fever, and the weight loss observed such as in this vignette [20-22]. Joint pain in the setting of an acute flair is more commonly observed. Moreover, the reports of multivessel coronary disease in the setting of GPA is scant in the literature. A few case reports in the literature tie coronary artery disease in the setting of GPA in patients, but not with multivessel disease warranting CABG $[23,24]$.

\section{Baseline risk factors in CAD}

Atherosclerosis is believed to be attributed as an underlying factor in roughly $50 \%$ of deaths in the Western world [25]. Given the high morbidity of disease, a commentary on independent underlying risk factors for cardiovascular disease in this patient should be introduced before a presentation of a unifying model encompassing inflammation as the etiology for a Major Adverse Cardiovascular Event (MACE) in the setting of GPA. Significant risk factors (although not an exhaustive list) of atherosclerosis include central adiposity (due to its propensity to promote deleterious metabolic pathways such as dysregulation of lipid metabolism), smoking which promotes microvascular dysfunction, and Type 2 Diabetes Mellitus (T2DM) in its propensity to promote global inflammation via reactive oxygen species formation [26-30]. This patient had a BMI of 28.7, categorizing him as overweight. Although it is well established that adiposity promotes atherosclerosis, there are no studies to date that explicitly delineate the differential risk stratification assessments of plaque burden by using only BMI to quantify the specific risk in this patient. A more accurate assessment of the morbidity associated with excess weight is giving way to scrutiny of adiposity distribution, namely through imaging modalities such as DEXA scan studies or measurement of anthropometric variables such as waist circumference or hip-to-waist ratios [31-34]. Specifically, a study by Park et al. was able to elucidate that the distribution of abdominal and thoracic fat tissue was one of the most puissant determinants of atherosclerotic risk [35]. The patient in this vignette also was moderately diabetic upon admission (HgbA1C, 7.8\%). Relative to the population of patients without vasculitis, a status of GPA confers a higher likelihood of T2DM as a comorbidity [36]. Whether this association is related to possible genetic linkage, vasculitis mediated damage to nodes of glycemic control (i.e. renal), or an independent association marked by lifestyle changes such as dietary indiscretion is unknown. T2DM exacerbates vasculopathy by aberrant hyperglycemic-induced mitochondrial metabolism, which induces inflammatory cascades affecting virtually every domain of atherogenesis through Reactive Oxygen Species (ROS) [37].

\section{Connecting GPA and CAD}

Cardiovascular disease in patients with GPA falls on a relatively wide spectrum. Previous reports in the literature note findings ranging from coronary arteritis to infiltrative heart disease $[38,39]$. Elucidation into the translational biology that connects the association between GPA and CAD have not been well mapped. GPA is marked by the presence of ANCAs that target serum proteinase 3 , which has been studied to promote neutrophilic degranulation. The differential affinity for these antibodies to cause degranulation in local environments which thereby result in the aforementioned clinical manifestations specific to each vasculitis as marked by the spectra of vasculitides is unknown, but it may be due to specific protein expressions along small-, medium- and large-sized vessels depending on the phenotypic architecture of these vessels that attract leukocytes to them [40]. In addition to damaging the endothelium of these vessels as previously mentioned, these processes also promote the uptake of oxidized low-density lipoprotein (LDL, oxLDL) by macrophages en route to foam cell formation, fatty streaks of atheromas, and finally, atherosclerosis. This pathway of inflammatory mediated damage with the aforementioned maladaptive cholesterol "plug" is illustrated in Figure 5.

\section{Inflammation \& GPA-tying the Knot}

The specific triggers for the induction of granulomatous inflammation and autoimmunity in GPA remain mostly unclear [4144]. However, the behavior of PR3 in patients with GPA may provide insight into the pathophysiology of this patient and pointing towards a working model of multivessel CAD. PR3-ANCA is present in roughly $50 \%$ of patients with localized GPA (that is, involving a solitary distribution of tissue, most commonly of ENT or renal distribution) while its presence can be appreciated in over $90 \%$ of cases that present with systemic GPA $[45,46]$. The levels of PR3-ANCA at remission are a puissant indicator of future relapse or prospective morbidity in patients with GPA, highlighting the focus of these antibodies and 
their role in PR3 signaling $[47,48]$. It has been demonstrated that PR3 is expressed constitutively on neutrophils via immunofluorescent studies of 89 patients with GPA [49]. In addition, there is evidence to support pathways showing PR3-ANCA promotes activation and degranulation in neutrophils primed with complement factors such as C5a in addition to cytokines (IL-1 and TNF- $\alpha$ ) mediated by this membrane-bound PR3 [50-52].

Studies using in-situ hybridization to locate the presence of IL-1 mRNA within coronary vessel walls have shown that this family of interleukins is upregulated both in patients with atherosclerotic disease as well as increased disease burden of CAD [53]. Additionally, IL-1 exhibits activity within the architecture of atheromas in human. Namely, it may induce procoagulant pathways important for prothrombotic sequelae of plaque disruption, an increase in adhesion molecules that bring leukocytes central to plaque architecture, and the production of monocyte chemoattractant protein 1 (MCP-1) [54-56] MCP-1 is especially important for the promotion of monocyte flux that promotes oxLDL consumption and the initiation of the foam cell in atherogenesis, and its antagonism or receptor targeting has been of recent interest in the mitigation of atherosclerotic disease progression [57,58]. Early studies using immunohistochemical staining have shown MCP-1 mRNA to be ubiquitous within the macrophages of atherosclerotic plaques in humans [59].

The other inflammatory cytokine discussed regarding the contents of granulated neutrophils is TNF- $\alpha$. At more advanced stages of atherosclerosis, TNF- $\alpha$ has been shown to promote plaque disruption via apoptosis, and may be one of the players implicated in the pathogenesis of MACE in patients with CAD. Namely, the notion of apoptotic macrophages contributes to an enlarging of the lipid core, and the death of smooth muscle cells may promote the architecture of the fibrous cap within a plaque [60-62]. These events ultimately favor plaque rupture. Moreover, TNF- $\alpha$ may also promote the initial step of atherogenesis, microvascular disruption via endothelial cell insult. Increased levels of TNF- $\alpha$ promote increased expression and activity or arginase, an enzyme that acts competitively against endothelial Nitric Oxide Synthase (eNOS), and subsequently decreasing the levels of Nitric Oxide (NO) within the endothelial cells by promoting aberrant endothelial cell function $[63,64]$. Moreover, a deficiency of eNOS attributable to pathologic TNF- $\alpha$ expression has the propensity to compromises endothelial innervation, resulting in loss of autonomic regulation and subsequently leading to a net contractile state amenable to hypertensive shear forces and further microvascular damage and cholesterol deposition [65].

A study by Krajewska et al. examined the serum levels of IL-32 and IL- 6 in patients with GPA, showing that concentrations of IL-32 were positively correlated with PR3-ANCA levels in GPA $(\mathrm{P}<0.0001)$ [66]. Increased IL-32 levels have been associated with macrophage lipid metabolism as well as metalloproteinase expression, the latter of which is implicated in plaque instability [67]. It has also been shown to be present in excess within the plaques of patients with CAD relative to healthy controls [68]. Moreover, IL-32 is associated with increased levels of the pro-atherosclerotic cytokine TNF- $\alpha$, which subsequently promotes increased IL-32 in an "inflammatory loop" [69]. This gives credence to the notion that IL-32, and by proxy, the status of GPA diagnosis does not just promote atherosclerotic plaque development but also sequelae related to MACE such as rupture and embolization through downstream molecular modulation.

However, the maintenance of plaque via inflammatory pathways are not the only culpable variables when elucidating the map from ANCA to atherosclerosis. Impaired immunomodulatory functions, namely via regulatory T-cells (Treg) offer a perspective into this perspective of GPA-mediated CAD. It has been demonstrated that Treg function is attenuated towards PR3-induced proliferation of T-cells, thus prompting inflammation [70-73]. This differential function is appreciated as an expanded Th17 population in patients with GPA [74,75]. An imbalance between Treg and Th17 expression and differential function has been widely studied as inducers of a CAD phenotype [76,77]. Although unclear, theories for this association and CAD are centered upon modulation of oxLDL metabolism, highlighting the propensity for ANCA vasculitides to not only promote the development of atherosclerotic plaques, but their initial development as well.

\section{Pharmacotherapy and plaques- what's the connection?}

While a discussion regarding the pathology of GPA and its overlap with $\mathrm{CAD}$ helps place context with regards to the physiological events leading up to this presentation, a discussion on pharmacotherapy and its role is essential for further insight. Cyclophosphamide and prednisone are a commonly used treatment regimen for ANCA-Associated Vasculitis (AAV). The mechanism of action of cyclophosphamide can be appreciated through its metabolite, phosphoramide mustard, which crosslinks DNA strands and promotes apoptosis of immune cells. This leads to an attenuation of the immune response via inhibition of the propensity for T-cell mediated cellular signaling cascades that interact with PR3 and initiate PR3-ANCA pathways. Moreover, prednisone both decreased systemic inflammation but also suppresses the migration of polymorphonuclear leukocytes, which diminishes the proclivity for neutrophil degranulation along vessels, thus inhibiting the initial vascular insult that may initiate atherosclerosis. However, while it is suggested that cyclophosphamide offers benefit in these patient populations via attenuation of the inflammatory processes that promote plaque progression and disruption, there also is a potential avenue for these medications to slightly decrease atherosclerotic burden in animal models [78].

\section{Conclusion}

While the buildup of plaque in the setting of CAD is classically associated with hypertension, diabetes, and external vascular insults such as derivative from smokes, the influence of autoimmune disease of vasculature cannot be ignored. Namely, vasculitis leading to oxidized LDL deposition and subsequent complicated CAD. Workup for vasculitis through an audit of autoimmune biomarkers remains viable options for diagnosing patients with previous signs of vascular compromise and otherwise ambiguous symptoms seen in our patient. Early diagnosis with the establishment of protocols balancing cardiovascular symptoms in conjunction with autoimmune profile with medical management in these patients should be considerations, as salvaging myocardial perfusion as soon as possible is one of the most puissant predictors of long-term morbidity in heart disease.

\section{References}

1. Máchal J, Pavkova-Goldbergova M, Hlinomaz O, Groch L, Vasku A. Patients with chronic three-vessel disease in a 15-year follow-up study: genetic and 
non-genetic predictors of survival. Medicine. 2014; 93: e278.

2. Zeng X, Chen J, Miller $\mathrm{YI}$, Javaherian K, Moulton KS. Endostatin binds biglycan and LDL and interferes with LDL retention to the subendothelia matrix during atherosclerosis. Journal of lipid research. 2005; 46: 1849-1859.

3. Chistiakov DA, Melnichenko AA, Orekhov AN, Bobryshev YV. How do macrophages sense modified low-density lipoproteins? International journal of cardiology. 2017; 230: 232-240.

4. Kalampogias A, Siasos G, Oikonomou E, Tsalamandris S, Mourouzis K Tsigkou $\mathrm{V}$, et al. Basic mechanisms in atherosclerosis: the role of calcium. Medicinal Chemistry. 2016; 12: 103-113.

5. Goikuria H, Vandenbroeck K, Alloza I. Inflammation in human carotid atheroma plaques. Cytokine \& growth factor reviews. 2018; 39: 62-70.

6. Arbab-Zadeh A, Fuster V. From detecting the vulnerable plaque to managing the vulnerable patient: JACC state-of-the-art review. Journal of the American College of Cardiology. 2019; 74: 1582-1593.

7. Suda A, Takahashi J, Hao K, Kikuchi Y, Shindo T, Ikeda S, et al. Coronary functional abnormalities in patients with angina and nonobstructive coronary artery disease. Journal of the American College of Cardiology. 2019; 74 2350-2360.

8. Niccoli G, Montone RA, Sabato V, Crea F. Role of allergic inflammatory cells in coronary artery disease. Circulation. 2018; 138: 1736-1748.

9. Rhew EY, Ramsey-Goldman R. Premature atherosclerotic disease in systemic lupus erythematosus-role of inflammatory mechanisms. Autoimmunity Reviews. 2006; 5: 101-105.

10. Kato $\mathrm{H}$, Inoue $\mathrm{O}$, Toshima $\mathrm{H}$, Kawasaki $\mathrm{T}$, Fujiwara $\mathrm{H}$, Watanabe $\mathrm{T}$. Adult coronary artery disease probably due to childhood Kawasaki disease. The Lancet. 1992; 340: 1127-1129.

11. Tadokoro N, Fujita T, Fukushima S, Simahara Y, Matsumoto Y, Yamashita $\mathrm{K}$, et al. Multiple Coronary Artery Bypass Grafting for Kawasaki DiseaseAssociated Coronary Artery Disease. The Annals of thoracic surgery. 2019 108: 799-805.

12. Madhavan MV, Coromilas EJ, Poterucha TJ, Weiner SD, Smith CR, Prasad $M$, et al. Repeat revascularization for severe recurrent coronary artery disease in a young woman with Takayasu arteritis. Case Reports. 2020; 2: 77-81.

13. Pan L, Du J, Liu J, Liao H, Liu X, Guo X, et al. Tocilizumab treatment effectively improves coronary artery involvement in patients with Takayasu arteritis. Clinical rheumatology. 2020; 39: 2369-2378.

14. Chai JT, McGrath S, Lopez B, Dworakowski R. Eosinophilic granulomatosis with polyangiitis (Churg-Strauss syndrome) masquerading as acute ST-elevation myocardial infarction with complete resolution after immunosuppressive therapy: a case report. European Heart Journal-Case Reports. 2018; 2: yty075.

15. Nandkeolyar S, Kim HB, Doctorian T, Stoletniy LN, Sandhu VK, Yu M, et al A case report of heart transplant for ischaemic cardiomyopathy from lupus coronary vasculitis. European Heart Journal: Case Reports. 2019; 3: 1-7.

16. Al-Hussain T, Hussein MH, Conca W, Al Mana H, Akhtar M. Pathophysiology of ANCA-associated vasculitis. Advances in anatomic pathology. 2017; 24 226-234.

17. McGeoch L, Carette S, Cuthbertson D. Cardiac involvement in granulomatosis with polyangiitis. The Journal of Rheumatology. 2015; 42: 1209-1212.

18. Berden A, Göçeroğlu A, Jayne D. Diagnosis and management of ANCA associated vasculitis. Bmj. 2012; 344

19. Wallace ZS, Lu N, Miloslavsky E, Unizony S, Stone JH, Choi HK. Nationwide trends in hospitalizations and in hospital mortality in granulomatosis with polyangiitis (Wegener's). Arthritis care \& research. 2017; 69: 915-921.

20. Comarmond C, Cacoub P. Granulomatosis with polyangiitis (Wegener) clinical aspects and treatment. Autoimmunity reviews. 2014; 13: 1121-1125.

21. Hassane HH, Beg MM, Siva C, Velázquez C. Co-presentation of giant cell arteritis and granulomatosis with polyangiitis: a case report and review of literature. The American journal of case reports. 2018; 19: 651-655.
22. Bohm M, Fernandez MIG, Ozen S, et al. Clinical features of childhood granulomatosis with polyangiitis (wegener's granulomatosis). Pediatric Rheumatology. 2014; 12: 1-5.

23. Mourguet M, Chauveau D, Faguer S. Increased ischemic stroke, acute coronary artery disease and mortality in patients with granulomatosis with polyangiitis and microscopic polyangiitis. Journal of autoimmunity. 2019; 96 : 134-141.

24. Luo Y, Xu J, Jiang C. Trends in the Inpatient Burden of Coronary Artery Disease in Granulomatosis with Polyangiitis: A Study of a Large National Dataset. The Journal of Rheumatology. 2020.

25. Pahwa R, Jialal I. StatPearls. Atherosclerosis Treasure Island (FL): StatPearls Publishing. 2019.

26. Roever LS, Resende ES, Diniz ALD. Abdominal obesity and association with atherosclerosis risk factors: the Uberlândia heart study. Medicine. 2016; 95 E1357.

27. Weber T, Lang I, Zweiker R. Hypertension and coronary artery disease: epidemiology, physiology, effects of treatment, and recommendations. Wiener klinische Wochenschrift. 2016; 128: 467-479

28. Gellert KS, Keil AP, Zeng D. Abstract P232: Modifying General and Central Adiposity: Estimated Effect on Population Burden of Coronary Heart Disease. The Atherosclerosis Risk in Communities (ARIC) Study. Circulation. 2017; 135: AP232-AP232.

29. Parsons C, Agasthi P, Mookadam F, Arsanjani R. Reversal of coronary atherosclerosis: role of life style and medical management. Trends in cardiovascular medicine. 2018; 28: 524-531.

30. Yahagi K, Kolodgie FD, Lutter C. Pathology of human coronary and carotid artery atherosclerosis and vascular calcification in diabetes mellitus. Arteriosclerosis, thrombosis, and vascular biology. 2017; 37: 191-204.

31. Neeland IJ, Grundy SM, Li X, Adams-Huet B, Vega GL. Comparison of visceral fat mass measurement by dual-X-ray absorptiometry and magnetic resonance imaging in a multiethnic cohort: the Dallas Heart Study. Nutrition \& diabetes. 2016; 6: e221-e221.

32. Vega GL, Adams-Huet B, Peshock R, Willett D, Shah B, Grundy SM Influence of body fat content and distribution on variation in metabolic risk The Journal of Clinical Endocrinology \& Metabolism. 2006; 91: 4459-4466.

33. Imahori Y, Mathiesen EB, Leon DA, et al. The contribution of obesity to carotid atherosclerotic plaque burden in a general population sample in Norway: the Troms $ø$ study. Atherosclerosis. 2018; 273: 15-20.

34. Rodriguez-Granillo GA, Reynoso E, Capunay C, Carpio J, Carrascosa P Pericardial and visceral, but not total body fat, are related to global coronary and extra-coronary atherosclerotic plaque burden. International journal of cardiology. 2018; 260: 204-210

35. Park S-J, Yang H-M, Seo K-W. The relationship between coronary atherosclerosis and body fat distribution measured using dual energy $\mathrm{X}$-ray absorptiometry. Atherosclerosis. 2016; 248: 190-195

36. Li L, Neogi T, Jick S. A cohort study of comorbidity in patients with granulomatosis with polyangiitis. Rheumatology. 2018; 57: 291-299.

37. Rendra E, Riabov V, Mossel DM, Sevastyanova T, Harmsen MC Kzhyshkowska J. Reactive Oxygen Species (ROS) in macrophage activation and function in diabetes. Immunobiology. 2019; 224: 242-253.

38. Morelli S, Di Castelmenardo AMG, Conti F. Cardiac involvement in patients with Wegener's granulomatosis. Rheumatology international. 2000; 19: 209 212

39. Florian A, Slavich M, Blockmans D, Dymarkowski S, Bogaert J. Cardiac involvement in granulomatosis with polyangiitis (Wegener granulomatosis) Circulation. 2011; 124: e342-e344.

40. Terrier B, Dechartres A, Deligny C. Granulomatosis with polyangiitis according to geographic origin and ethnicity: clinical-biological presentation and outcome in a French population. Rheumatology. 2017; 56: 445-450.

41. Sarraf $P$, Sneller MC. Pathogenesis of Wegener's granulomatosis: current concepts. Expert reviews in molecular medicine. 2005; 7: 1-19. 
42. Greco A, Marinelli C, Fusconi M. Clinic manifestations in granulomatosis with polyangiitis. International journal of immunopathology and pharmacology. 2016; 29: 151-159.

43. Deng $\mathrm{S}$, Xing $\mathrm{Y}$, Wang $\mathrm{H}$, Kong $X$. Relapsing granulomatosis with polyangiitis provoked by trauma: A case report. Medicine. 2019; 98: E14189.

44. Gadola SD, Gross WL. The renaissance of granulomatous inflammation in AAV. Nature Reviews Rheumatology. 2012; 8: 74-76.

45. Csernok E, Lamprecht $P$, Gross WL. Diagnostic significance of ANCA in vasculitis. Nature Clinical Practice Rheumatology. 2006; 2: 174-175.

46. Holle JU, Gross WL, Latza U. Improved outcome in 445 patients with Wegener's granulomatosis in a German vasculitis center over four decades. Arthritis \& Rheumatism. 2011; 63: 257-266.

47. Terrier B, Pagnoux C, Perrodeau É, et al. Long-term efficacy of remissionmaintenance regimens for ANCA-associated vasculitides. Annals of the Rheumatic Diseases. 2018; 77: 1150-1156.

48. Morgan MD, Szeto M, Walsh M. Negative anti-neutrophil cytoplasm antibody at switch to maintenance therapy is associated with a reduced risk of relapse. Arthritis research \& therapy. 2017; 19: 1-7.

49. Rarok AA, Stegeman CA, Limburg PC, Kallenberg CGM. Neutrophil membrane expression of proteinase 3 (PR3) is related to relapse in PR3ANCA-associated vasculitis. Journal of the American Society of Nephrology 2002; 13: 2232-2238.

50. Brilland B, Garnier A-S, Chevailler A, Jeannin P, Subra J-F, Augusto J-F. Complement alternative pathway in ANCA-associated vasculitis: Two decades from bench to bedside. Autoimmunity reviews. 2020; 19: 102424

51. Hess C, Kemper C. Complement-mediated regulation of metabolism and basic cellular processes. Immunity. 2016; 45: 240-254.

52. Hao J, Meng L-Q, Xu P-C, Chen M, Zhao M-H. p38MAPK, ERK and PI3K signaling pathways are involved in C5a-primed neutrophils for ANCAmediated activation. PloS One. 2012; 7: e38317.

53. Galea J, Armstrong J, Gadsdon P, Holden H, Francis SE, Holt CM Interleukin-1 $\beta$ in coronary arteries of patients with ischemic heart disease. Arteriosclerosis, thrombosis, and vascular biology. 1996; 16: 1000-1006.

54. Grover SP, Mackman N. Tissue factor: an essential mediator of hemostasis and trigger of thrombosis. Arteriosclerosis, thrombosis, and vascular biology. 2018; 38: 709-725.

55. Buckley LF, Abbate A. Interleukin-1 blockade in cardiovascular diseases: a clinical update. European heart journal. 2018; 39: 2063-2069.

56. Hartman MHT, Groot HE, Leach IM, Karper JC, van der Harst P. Translationa overview of cytokine inhibition in acute myocardial infarction and chronic heart failure. Trends in cardiovascular medicine. 2018; 28: 369-379.

57. Xia M, Sui Z. Recent developments in CCR2 antagonists. Expert opinion on therapeutic patents. 2009; 19: 295-303.

58. Maguire EM, Pearce SWA, Xiao Q. Foam cell formation: A new target for fighting atherosclerosis and cardiovascular disease. Vascular Pharmacology. 2019; 112: 54-71.

59. Nelken NA, Coughlin SR, Gordon D, Wilcox JN. Monocyte chemoattractant protein-1 in human atheromatous plaques. The Journal of clinical investigation. 1991; 88: 1121-1127.

60. Linton MF, Babaev VR, Huang J, Linton EF, Tao H, Yancey PG. Macrophage apoptosis and efferocytosis in the pathogenesis of atherosclerosis. Circulation Journal. 2016; 80: 2259-2268.

61. Tang M, Fang J. TNF- $\alpha$ regulates apoptosis of human vascular smooth muscle cells through gap junctions. Molecular medicine reports. 2017; 15 1407-1411.
62. Hansson GK, Libby P, Tabas I. Inflammation and plaque vulnerability. Journal of internal medicine. 2015; 278: 483-493.

63. Zhu C, Yu Y, Montani J-P, Ming X-F, Yang Z. Arginase-I enhances vascular endothelial inflammation and senescence through eNOS-uncoupling. BMC Research Notes. 2017; 10: 82.

64. Machado-Oliveira G, Ramos C, Marques ARA, Vieira OV. Cell Senescence, Multiple Organelle Dysfunction and Atherosclerosis. Cells. 2020; 9: 2146

65. Xia N, Forstermann U, Li H. Implication of eNOS uncoupling in cardiovascular disease. Reactive Oxygen Species. 2017; 3: 38-46.

66. Krajewska J, Kościelska-Kasprzak K, Krajewski W, Morawski K. Serum levels of interleukin-32 and interleukin-6 in granulomatosis with polyangiitis and microscopic polyangiitis: association with clinical and biochemical findings. European Cytokine Network. 2019; 30: 151-159.

67. Heinhuis B, Popa CD, van Tits BLJH. Towards a role of interleukin-32 in atherosclerosis. Cytokine. 2013; 64: 433-440

68. Yang Z, Shi L, Xue Y. Interleukin-32 increases in coronary arteries and plasma from patients with coronary artery disease. Clinica Chimica Acta. 2019; 497: 104-109.

69. Damen MSMA, Popa CD, Netea MG, Dinarello CA, Joosten LAB Interleukin-32 in chronic inflammatory conditions is associated with a higher risk of cardiovascular diseases. Atherosclerosis. 2017; 264: 83-91.

70. Rimbert M, Hamidou M, Braudeau C, et al. Decreased numbers of blood dendritic cells and defective function of regulatory $T$ cells in antineutrophil cytoplasmic antibody-associated vasculitis. PloS one. 2011; 6: e18734.

71. Martinez Valenzuela L, Bordignon Draibe J, Fulladosa Oliveras X, Bestard Matamoros O, Cruzado Garrit JM, Torras Ambrós J. T-lymphocyte in ANCAassociated vasculitis: what do we know? A pathophysiological and therapeutic approach. Clinical Kidney Journal. 2019; 12: 503-511.

72. Klapa S, Mueller A, Csernok E. Lower numbers of FoxP3 and CCR4 coexpressing cells in an elevated subpopulation of CD4+CD25high regulatory $T$ cells from Wegener's granulomatosis. Clinical \& Experimental Rheumatology. 2010; 28: S72-S80.

73. von Borstel A, Sanders JS, Rutgers A, Stegeman CA, Heeringa P, Abdulahad $\mathrm{WH}$. Cellular immune regulation in the pathogenesis of ANCA-associated vasculitides. Autoimmunity reviews. 2018; 17: 413-421.

74. Wilde B, Thewissen M, Damoiseaux J, et al. Th17 expansion in granulomatosis with polyangiitis (Wegener's): the role of disease activity, immune regulation and therapy. Arthritis research \& therapy. 2012; 14: R227.

75. Cosmi L. Th17 and Treg lymphocytes as cellular biomarkers of disease activity in Granulomatosis with Polyangiitis. European Journal of Immunology. 2017; 47: 633-636.

76. Potekhina AV, Pylaeva E, Provatorov S. Treg/Th17 balance in stable CAD patients with different stages of coronary atherosclerosis. Atherosclerosis. 2015; 238: 17-21.

77. Wang Z, Lee J, Zhang Y. Increased Th17 cells in coronary artery disease are associated with neutrophilic inflammation. Scandinavian Cardiovascular Journal. 2011; 45: 54-61.

78. Sato-Okabayashi Y, Isoda K, Heissig B. Low-dose oral cyclophosphamide therapy reduces atherosclerosis progression by decreasing inflammatory cells in a murine model of atherosclerosis. IJC Heart \& Vasculature 2020; 28. 100529 . 